\title{
O apego ao lugar no contexto dos estudos pessoa-ambiente: práticas de pesquisa'
}

\author{
Place attachment in the context of person-environment \\ studies: research practices
}

\author{
Maíra Longhinotti FELIPPE \\ Ariane KUHNEN ${ }^{3}$
}

\section{Resumo}

Objeto de estudo de disciplinas interessadas nas relações humano-ambientais, o apego ao lugar traduz-se pelo vínculo afetivo estabelecido entre pessoas e cenários físicos. Com o objetivo de avaliar as práticas de investigação do apego ao lugar quanto ao modo como se relacionam às características dos estudos da Psicologia Ambiental, fez-se análise da produção de artigos científicos relacionados ao tema, disponibilizados nas bases de dados Science Direct e Sage. Examinaram-se aspectos como a complexidade do objeto de estudo, a multidisciplinaridade, as perspectivas teóricas, os paradigmas de investigação e a abordagem multimetodológica. Os resultados da análise demonstraram que a pesquisa do apego ao lugar parece ainda evitar a triangulação metodológica proposta para os estudos pessoa-ambiente, mas, de modo geral, apontaram proximidades quanto a sua investigação e à produção de conhecimento da Psicologia Ambiental.

Unitermos: Comportamento de apego. Epistemologia. Metodologia. Psicologia ambiental.

\begin{abstract}
As the study target on areas interested in human-environmental relationships, place attachment is defined by theemotional bond established between people and physical settings. In order to evaluate the research practices of place attachment with regard to how they relate to the characteristics of the studies of Environmental Psychology, the scientific production related to this area, available in Science Direct and Sage databases was analyzed. The complexity of the study object, its multidisciplinarity, theoretical perspectives, research paradigms and the multimethodological approach were analyzed. Analysis showed that place attachment researches still seem to avoid the methodological triangulation proposed for person-environment studies; however, in general, they showed proximity as regards their investigation and production of knowledge of Environmental Psychology.
\end{abstract}

Uniterms: Attachment behavior. Epistemology. Methodology. Environmental psychology.

O interesse pela pesquisa sistemática dos laços afetivos que as pessoas estabelecem com lugares decorre da percepção do papel que esses laços possuem em contribuir para a definição e a qualificação da identidade pessoal, instaurar o sentido de pertencimento a um lugar e promover a apropriação e o cuidado ambien-

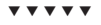

1 Apoio: Coordenação de Aperfeiçoamento de Pessoal de Nível Superior.

2 Università degli Studi di Ferrara, Dipartimento di Architettura. Ferrara, Itália.

3 Universidade Federal de Santa Catarina, Centro de Filosofia e Ciências Humanas, Programa de Pós-Graduação em Psicologia, Departamento de Psicologia. Campus Universitário, Trindade, 88040-900, Florianópolis, SC, Brasil. Correspondência para/Correspondence to: A. KUHNEN. E-mail: <ariane@cfh.ufsc.br>. 
tais (Giuliani, 2003; Proshansky, Fabian \& Kaminoff, 1983; Speller, 2005). A pesquisa dedicada ao tema tem ampliado significativamente seu âmbito de investigação e encontrado variados campos de aplicação. Vínculos emocionais com lugares têm sido relacionados a comprometimento e comportamento pró-ambientais (Bonaiuto, Carrus, Martorella \& Bonnes, 2002; Brown, Reed \& Harris, 2002; Buijs, 2009; Jorgensen \& Stedman, 2006; Ryan, 2005; Walker \& Ryan, 2008); cuidados com o local de moradia e vigilância para a manutenção da ordem em comunidades (B.B. Brown, Perkins \& G. Brown, 2003, 2004; G. Brown, B.B. Brown \& Perkins, 2004); mobilização para o planejamento ambiental comunitário, engajamento e controle social (Brown et al., 2003; Brown et al., 2004; Kelly \& Hosking, 2008; Lewicka, 2005; Manzo \& Perkins, 2006; Wakefield, Elliott, Cole \& Eyles, 2001; Walker \& Ryan, 2008), lealdade para com destinos turísticos (Gross \& Brown, 2006, 2008; Hailu, Boxall \& McFarlane, 2005; Hwang, Lee \& Chen, 2005; A. Yuksel, F. Yuksel \& Bilim, 2010) e estabelecimentos comerciais (Allard, Babin \& Chebat, 2009; Rosenbaum, Ward, Walker \& Ostrom, 2007).

A afetividade envolvendo lugares comparece, com maior ou menor destaque, em estudos de diferentes grupos temáticos da Psicologia Ambiental (PA). Citam-se as pesquisas sobre comportamento socioespacial humano, conhecimento ambiental, ambientes e populações específicas, avaliação e planejamento ambiental, comportamento ecologicamente responsável, percepção social de riscos ambientais e gestão ambiental, segundo classificação apresentada por Pinheiro (2003), ao caracterizar a pesquisa nos estudos pessoa-ambiente.

As considerações propostas a seguir avaliam de que forma a pesquisa do apego ao lugar tem se relacionado com os modos de ser e fazer característicos dos estudos da PA. Observaram-se aspectos como a complexidade do objeto estudado, a multidisciplinaridade, as perspectivas teóricas, os paradigmas de investigação e a abordagem multimetodológica, categorias de análise importantes para a caracterização do campo de estudo das relações mútuas estabelecidas entre ambiente e comportamento humano.
No que diz respeito à complexidade do objeto estudado, este trabalho pretende analisar se as definições do fenômeno, bem como dos indicadores e instrumentos de medida de apego ao lugar, oferecem sinais da complexidade que caracteriza as componentes comportamentais e ambientais dos estudos de PA. Quanto à multidisciplinaridade, objetiva verificar que departamentos disciplinares comparecem na pesquisa de apego ao lugar e se tal quadro é condizente com a variedade de formação dos pesquisadores da PA apontada pela literatura. Almeja igualmente comparar as perspectivas teóricas e os paradigmas de investigação utilizados, caracterizando e discutindo os estudos de apego ao lugar e as tendências em PA, segundo uma perspectiva teórica individualista, interacionista ou transacionalista, e um paradigma de investigação individualista-molecular ou social-molar. Por fim, pretende caracterizar a abordagem metodológica da pesquisa de apego ao lugar, confrontando-a com o enfoque de triangulação proposto para a PA.

\section{A afetividade nos estudos pessoa-ambiente}

O apego ao lugar pode ser definido como o vínculo emocional firmado com cenários físicos, envoLvendo sentimentos derivados da experiência espacial real ou esperada (Brown \& Perkins, 1992; Giuliani, 2004; Shumaker \& Taylor, 1983). Ao discutir sobre o conjunto de cognições de valência positiva e negativa elaboradas pelos sujeitos acerca do espaço físico, cognições estas a que chamaram identidade de lugar ${ }^{4}$, Proshansky, Fabian e Kaminoff (1983, p.76) postularam que o apego ao lugar"indubitavelmente ocorre naqueles indivíduos cuja identidade de lugar envolve cognições positivamente valorizadas de uma ou alguma combinação desses contextos, que de longe pesam mais que o número de cognições negativamente valorizadas". 0 conceito pressupõe que não há relação de afetividade humana que não esteja de alguma forma relacionada a aspectos de lugar, e considera, de modo semelhante ao que a teoria do apego (Bowlby, 1979/1997) considerou

\section{$\boldsymbol{\nabla} \boldsymbol{\nabla} \boldsymbol{\nabla}$}

4 Proshansky et al. (1983) afirmaram que a identidade de lugar é uma subestrutura ou um aspecto da identidade pessoal que se relaciona a contextos físicos. Segundo Speller (2005), entretanto, essa concepção merece ser ampliada ao se considerar que todo aspecto da identidade pessoal - e não somente uma 
para a relação interpessoal, a existência de vínculos emocionais envolvendo a dimensão física do ambiente.

Para Giuliani (2004), embora haja analogias evidentes entre a teoria do apego e a noção de apego ao lugar, este último é de natureza específica e distingue-se, portanto, de outras formas de afeto. Caracteriza-se, à semelhança dos laços afetivos entre pessoas (Ainsworth, 1989), pelo vínculo relativamente duradouro entre pessoa e ambiente, pela constituição de importância deste último em razão de sua singularidade, pelo desejo de proximidade ao lugar, pelo sentimento de segurança e conforto através do contato, e sofrimento em função da separação (Giuliani, 2004). Entretanto, é preciso observar que, embora a comparação com laços intrapessoais pareça ser útil, diferenças podem existir no que diz respeito à função psicológica de cada tipo de laço, às características do objeto de apego, ao comportamento manifesto, ao modo como o laço se forma, desaparece ou se transforma e a suas consequências psicológicas (Giuliani, 2003). Enquanto a teoria do apego procura as regularidades do comportamento de vinculação, a noção de apego ao lugar sublinha a diversidade dos laços entre indivíduos de diferentes grupos e culturas, e em diferentes estágios do ciclo de vida (Speller, 2005).

A primeira referência clara aos laços afetivos com lugares foi feita por Fried em 1963, na obra Grieving for a Lost Home, um estudo sobre a deslocação forçada da moradia (Giuliani, 2004). Fried percebeu que o afastamento do lugar produzia reações semelhantes à perda de um ente próximo e atribuiu esse sentimento a uma interrupção do sentido de continuidade dos moradores, pela fragmentação da identidade espacial e comunitária. Nessa mesma época, o debate acerca das diferenças entre as noções de espaço e lugar intensificou-se, em razão das massificações de realojamentos características das reestruturações urbanas do pós-guerra, que colocaram em questão a qualidade dos espaços produzidos (Speller, 2005). Enquanto a noção de espaço representava apenas um conjunto de positivações acerca do ambiente físico, o lugar constituía-se como o espaço imbuído do significado elaborado pelos seus utilizadores. A oposição espaço versus lugar pôs-se em cena no contexto de surgimento e desenvolvimento da PA. Essa área vem a se ocupar da pesquisa das relações psicológicas que ocorrem entre as pessoas e seus entornos.
Para avaliar a pesquisa do apego ao lugar quanto ao modo como se relaciona às características dos estudos de PA, fez-se análise da produção de artigos científicos relacionados ao tema, disponibilizados nas bases de dados Science Directe Sage, que reúnem, entre outros, periódicos voltados às áreas de ciências sociais e humanas. Dentre estes estão duas importantes publicações dedicadas aos estudos pessoa-ambiente: Environment \& Behavior, e Journal of Environmental Psychology. Resultados significativos da pesquisa também puderam ser encontrados em outros 17 periódicos, a saber: Anthropological Theory, Applied Geography, European Review of Applied Psychology, Habitat International, Health \& Place, International Social Work, Journal of Aging Studies, Journal of Business Research, Journal of Economic Psychology, Journal of Environmental Management, Journal of Planning Literature, Journal of Retailing and Consumer Services, Journal of Service Research, Landscape and Urban Planning, Organization \& Environment, Social Science \& Medicine e Tourism Management. A seguir, são apresentados alguns resultados da pesquisa, no que diz respeito ao grupo temático a que pertence o estudo, ao âmbito de investigação relacionado ao conceito de apego ao lugar e ao departamento de origem dos pesquisadores (Quadro 1).

A seleção de artigos ocorreu no mês de abril de 2009, identificando 46 trabalhos que incluíam a expressão place attachment em suas palavras-chave, ou seja, possuíam interesse específico sobre laços afetivos com lugares. Não houve limitação de intervalo de tempo para a pesquisa. A produção do conhecimento sobre apego ao lugar, dessa forma, permitiu avaliar sua investigação quanto a dimensões características dos estudos em PA: a complexidade do objeto estudado, a multidisciplinaridade, as perspectivas teóricas, os paradigmas de investigação e a abordagem multimetodológica.

\section{A complexidade do objeto estudado}

Para Günther, Elali e Pinheiro (2008), o conjunto pouco homogêneo de áreas de estudo e a variedade de formação dos pesquisadores na PA são reflexos de uma complexidade característica das componentes comportamentais e ambientais dos estudos pessoa-ambiente e da interação entre eles. Pode-se hipotetizar, inclusive, que é também devido à complexidade de seu objeto 
Quadro 1. Resultados da pesquisa de periódicos com interesse nas relações de apego ao lugar, publicados nas bases de dados Science Direct e Sage.

\begin{tabular}{|c|c|c|}
\hline Grupo temático & Âmbitos de investigação & Departamentos de origem \\
\hline Comportamento sócio-espacial humano & $\begin{array}{l}\text { Apropriação } \\
\text { Controle e coesão social } \\
\text { Cuidado ambiental } \\
\text { Dupla residência } \\
\text { Ecologia de comunidades } \\
\text { Impacto do turismo } \\
\text { Laços sociais } \\
\text { Movimentos sociais } \\
\text { Territorialidade } \\
\text { Transição à universidade }\end{array}$ & $\begin{array}{l}\text { Administração } \\
\text { Agricultura } \\
\text { Ambiente e Comportamento } \\
\text { Ambiente e Saúde } \\
\text { Aprendizagem } \\
\text { Comportamento Organizacional } \\
\text { Estudos Urbanos } \\
\text { Geografia } \\
\text { Geologia } \\
\text { Psicologia Ambiental } \\
\text { Psicologia Social } \\
\text { Sistemas Sustentáveis }\end{array}$ \\
\hline Conhecimento ambiental & Estabilidade do apego & $\begin{array}{l}\text { Ciência Florestal } \\
\text { Psicologia }\end{array}$ \\
\hline Ambientes especificos & $\begin{array}{l}\text { Áreas públicas urbanas } \\
\text { Contextos recreacionais } \\
\text { Escolas } \\
\text { Espaços comerciais } \\
\text { Festivais religiosos } \\
\text { Parques naturais } \\
\text { Shoppings }\end{array}$ & $\begin{array}{l}\text { Administração } \\
\text { Arquitetura } \\
\text { Ciência Florestal } \\
\text { Design } \\
\text { Economia Rural } \\
\text { Engenharia Civil } \\
\text { Hotelaria e Turismo } \\
\text { Marketing } \\
\text { Psicologia do Desenvolvimento } \\
\text { Recursos Naturais } \\
\text { Sociologia } \\
\text { Turismo e Recreação } \\
\text { Urbanismo }\end{array}$ \\
\hline Populações específicas & $\begin{array}{l}\text { Idosos } \\
\text { Nativos e não nativos }\end{array}$ & $\begin{array}{l}\text { Antropologia } \\
\text { Ciências da Saúde } \\
\text { Ciências do Comportamento } \\
\text { Geografia } \\
\text { Psicobiologia } \\
\text { Psicologia Cognitiva } \\
\text { Psicologia Social } \\
\text { Sociologia }\end{array}$ \\
\hline Avaliação e planejamento ambiental & $\begin{array}{l}\text { Relocação da moradia } \\
\text { Significado ambiental }\end{array}$ & $\begin{array}{l}\text { Arquitetura } \\
\text { Psicologia } \\
\text { Recursos Naturais }\end{array}$ \\
\hline Comportamento ecologicamente responsável & $\begin{array}{l}\text { Atividades recreacionais } \\
\text { Preservação de paisagens }\end{array}$ & $\begin{array}{l}\text { Arquitetura da Paisagem } \\
\text { Ciências Cognitivas } \\
\text { Planejamento Regional } \\
\text { Psicologia Social } \\
\text { Recreação e Turismo } \\
\text { Recursos Naturais } \\
\text { Sociologia Rural }\end{array}$ \\
\hline Percepção social de riscos ambientais & $\begin{array}{l}\text { Desastres naturais } \\
\text { Regiões de conflitos }\end{array}$ & $\begin{array}{l}\text { Arquitetura } \\
\text { Ciências da Saúde } \\
\text { Sociologia }\end{array}$ \\
\hline Gestão ambiental & Recuperação de mananciais & Urbanismo \\
\hline
\end{tabular}

612 
de estudo que a PA tenha hoje à sua disposição, como observou Valera (1996), um considerável número de definições. Para o autor, são razões para tal imprecisão: a difícil delimitação da área, face à sua localização fronteiriça a outras disciplinas; a sua história muito recente, visto que a PA adquiriu caráter próprio como disciplina apenas nos anos 1960; a sua natureza aplicada, condição que possivelmente não contribui para uma reflexão sobre suas bases teóricas; e, por fim, a multiplicidade de âmbitos de sua aplicação, que dificulta o estabelecimento de uma visão unitária de objeto de estudo.

Condições semelhantes puderam ser observadas na pesquisa de laços afetivos com lugares. A grande variedade de indicadores de ocorrência do fenômeno, observada nos artigos científicos selecionados, bem como a imprecisão nas definições utilizadas para tais indicadores e a ausência de padrão nas escalas de medida, inserem a pesquisa de apego ao lugar no contexto da complexidade dos fatos humanos e da PA. Giuliani (2003) comentou que a variedade de termos utilizados para fazer referência a apego ao lugar - enraizamento,

Quadro 2. Conceitos relacionados à ocorrência de apego ao lugar.

\begin{tabular}{ll}
\hline Indicadores de apego ao lugar \\
\hline 1. & Conforto \\
2. & Conhecimento do lugar \\
3. & Desejo de defender o lugar \\
4. & Desejo de proximidade e/ou envolvimento \\
5. & Dificuldades para substituição do local \\
6. & Felicidade \\
7. & Grau de atração \\
8. & Grau de cuidado com o lugar \\
9. & Grau de influência do lugar sobre os acontecimentos \\
10. & Mobilidade para a interação social \\
11. & Percepção de controle e possibilidade de ação \\
12. & Prazer \\
13. & Preferência \\
14. & Satisfação de interesses e necessidades \\
15. & Segurança \\
16. & Sensação de dependência \\
17. & Sentido de lar \\
18. & Sentimento de enraizamento \\
19. & Sentimento de identificação \\
20. & Sentimento de orgulho pelo lugar \\
21. & Sentimento de perda e/ou deslocamento pela separação \\
22. & Sentimento de pertencimento \\
23. & Sentimento de propriedade \\
\hline
\end{tabular}

pertencimento, afiliação, apropriação, compromisso, investimento, dependência, por exemplo - parece indicar uma imprecisão característica na identificação do fenômeno. Os conceitos relacionados à ocorrência de apego ao lugar verificados nos estudos são relacionados no Quadro 2.

Também na pesquisa de apego ao lugar observam-se as condições apresentadas por Valera (1996) para justificar a imprecisão de conceitos. A investigação do objeto compartilhada por grande diversidade de áreas profissionais, nem sempre operando em conjunto, e a natureza aplicada da pesquisa, voltada a uma multiplicidade de enfoques, como se pode observar nos dados apresentados pelo Quadro 1, possivelmente contribuem para a indefinição e a ausência de uniformidade nos critérios utilizados, bem como para a pouca valorização de pesquisa teórica que auxilie na definição desses mesmos critérios.

O fator tempo pode, igualmente, colaborar para essa condição. Como relata Giuliani (2003), depois de Fried e dos geógrafos Tuan, Relph e Buttimer nos anos 1970, o estudo do apego ao lugar compareceu como tópico de interesse primário somente na década de 1990. De fato, as pesquisas publicadas nos periódicos pesquisados datam dos últimos 15 anos, observando-se maior ocorrência de publicações de 2004 a 2009 (40 artigos), pouca ocorrência de 2000 a 2003 (5 artigos), e apenas uma publicação no período de 1995 a 1999.

\section{A multidisciplinaridade}

Com relação ao aspecto da multidisciplinaridade, também a investigação do apego ao lugar parece compartilhar o modo de ser da pesquisa em PA. Fruto da complexidade dos fatores envolvidos, a pesquisa multidisciplinar está na gênese dos estudos pessoa-ambiente. Verificou-se, pois, que 36 dos 46 artigos encontrados foram produzidos por departamentos externos à Psicologia. É oportuno lembrar que todos discutiam diretamente o conceito de apego que se estabelece com lugares. A diversidade de pesquisadores envolvidos é condizente com a descrição como comenta Pinheiro (2003), acerca dos grupos formadores da $\mathrm{PA}^{5}$, externos e internos à Psicologia. Assim, estiveram

\section{QVVV}

5 A Psicologia Ambiental recebeu influência de grupos disciplinares internos e externos à Psicologia. Da vertente interna, tem-se a Psicologia Social e a Psicologia da Percepção; da vertente externa, destacam-se a Arquitetura e o Planejamento Ambiental, a Geografia e as Ciências Biológicas (Pinheiro, 2003). 
presentes nos artigos pesquisados os seguintes departamentos: administração; agricultura e ecossistemas sustentáveis; antropologia; ciências ambientais; ciências da saúde; geografia; geologia; planejamento e arquitetura; psicobiologia; sociologia; recursos naturais e turismo; além da psicologia cognitiva, do desenvolvimento, organizacional e social. Entretanto, apenas cerca de um terço dos artigos pesquisados (14 artigos) foram produto da investigação conjunta de dois ou mais departamentos disciplinares. Desafios são lançados, por isso, no sentido da maior cooperação entre as disciplinas (Elali, 1997), para que se garanta a chamada interdisciplinaridade, que supera as diferenças de linguagem, enfoques, metodologia e perspectivas teóricas.

\section{As perspectivas teóricas e os paradigmas de investigação}

Possivelmente porque tanto tem origem em grupos disciplinares diversos, como é subárea da Psicologia - apontada por críticos como uma ciência incompletamente formada (Sève, 1979), por sua imaturidade para definições de objeto, método, conceitos de base e leis fundamentais de desenvolvimento - a PA apresenta, segundo Valera (1996), uma multiplicidade de orientações teóricas e tradições de investigação. Valera discutiu as diversas formas de análise da relação pessoa-ambiente observadas na Psicologia Ambiental e, com base no modelo de Altman e Rogoffe de Saegert e Winkel, apresentou, respectivamente, as perspectivas teóricas e os paradigmas de investigação da disciplina.

Quanto às orientações teóricas, o autor apontou as perspectivas individualista, interacionista e sistêmica/transacionalista. A perspectiva individualista tem a unidade de análise centrada na pessoa, em detrimento das variáveis ambientais, que possuem papel secundário. Na orientação interacionista, por sua vez, a unidade de análise é a pessoa e o ambiente, como elementos distintos. Considera-se aí que há interação (influência) entre os dois componentes, sendo o comportamento (variável dependente) função do ambiente (variável independente) e da pessoa (variável mediadora). Já a perspectiva sistêmica/transacionalista considera como unidade de análise a pessoa inserida no ambiente. Nesse caso, ambos formam um sistema integrado de interrelações, com ênfase do todo em relação às partes. Essa que pessoa e ambiente definem-se e transformam-se mutuamente. Segundo Valera (1996), as duas primeiras perspectivas, mais tradicionais, têm sido substituídas pela perspectiva sistêmica/transacionalista, mais atual.

Essa tendência pode ser observada quanto à análise de apego ao lugar nos artigos pesquisados. Apenas investigações em contextos turístico-recreacionais e comerciais não deram ênfase à perspectiva de sistema e de bidirecionalidade, podendo ser classificadas tão somente como interacionistas. As demais pesquisas demonstraram uma compreensão sistêmica do fenômeno - como as que discutiam laços sociais e territorialidade - e uma abordagem bidirecional, especialmente evidente nos estudos que envolvem comprometimento e comportamento pró-ambiental.

Quanto aos paradigmas de investigação da PA, apontados por Valera (1996), nota-se a constituição de dois grupos: o paradigma individualista-molecular e o paradigma social-molar. Essas perspectivas se referem a níveis de análise da relação pessoa-ambiente, do mais individual ao mais social. As categorias não são excludentes entre si e há uma tendência atual de interagir níveis de análise distintos, com ênfase no social. Da mesma forma, essa tendência foi observada na pesquisa sobre apego ao lugar. De modo geral, as perspectivas individualistas-moleculares coincidiram com as interacionistas, destacando-se a relação entre a capacidade do entorno em satisfazer necessidades e a seleção de melhores ambientes. Em outra mão, a perspectiva social-molar coincidiu com a sistêmica/transacionalista, em que a pessoa pode ser vista como agente social que constrói significados de lugar modulados pela cultura e pela sociedade.

\section{A abordagem multimetodológica}

Por fim, analisou-se o aspecto da abordagem multimetodológica, que avança em direção à multiplicidade de aspectos caracterizadores de um problema de pesquisa. A adoção de multimétodos produz informações complementares da situação e é indispensável à validade dos resultados obtidos, já que minimiza os vieses próprios de procedimentos que avaliam um único aspecto do problema (Günther et al., 2008). A triangulação metodológica é proposta para a PA e parte do pressuposto de que cada método, por suas qualidades 
e limitações intrínsecas, possibilita elucidar apenas fragmentos do fenômeno estudado, sendo necessários, portanto, caminhos diversos para se alcançar a compreensão de um único objeto.

Assim, o método experimental - que permite inferências causais, mas considera situações artificiais de pesquisa - pode ser complementar ao método observacional - que prima pelo realismo, mas não possui as vantagens do controle - e ao método interrogativo do levantamento de dados, que permite análises estatísticas substanciais, apesar dos vieses próprios da interação social. Técnicas verbais podem complementar as não verbais, assim como análises quantitativas aprofundam as possibilidades de investigação das qualitativas e vice-versa.

No que se refere à pesquisa do apego ao lugar, no entanto, houve diferenças no que diz respeito à metodologia esperada. A quase totalidade dos estudos restringiu o delineamento metodológico às técnicas de questionário e entrevista semiestruturada, com utilização de escala de valores e análise de conteúdo. Poucas referências foram feitas ao uso de mapas mentais (uma ocorrência), fotografias (duas ocorrências), observação participante (uma ocorrência) e pesquisa documental somada a diário de campo (uma ocorrência). Acredita-se que o fato se deve à grande participação, como visto, das ciências sociais aplicadas, que utilizam largamente os questionários e as entrevistas em suas investigações. A literatura, entretanto, aponta que a afetividade tem sido investigada com êxito através do uso de desenhos, fotografias, autobiografias e mapas cognitivos (Günther et al., 2008). Também as técnicas de observação de vestígios ambientais e mapeamento comportamental ${ }^{6}$ podem oferecer o suporte e as vantagens do método observacional.

\section{Considerações Finais}

Os apontamentos considerados avaliaram de que forma a pesquisa do apego ao lugar tem se relacionado com os modos de ser e fazer característicos dos estudos da PA. A análise da produção de artigos científicos relacionados ao tema do apego permitiu comparar as práticas de ambos os grupos, no que diz respeito à complexidade do objeto estudado, à multidisciplinaridade, às perspectivas teóricas e paradigmas de investigação, bem como à abordagem multimetodológica. Observaram-se similaridades quanto aos três primeiros aspectos e diferenças com relação ao último, pois o estudo do apego ao lugar parece ainda evitar a triangulação metodológica. Os resultados demonstram a dificuldade imposta pela convergência de métodos, mas, de modo geral, apontam proximidades quanto às práticas de pesquisa do apego ao lugar no contexto da PA.

Não foram encontrados artigos de produção nacional para o critério adotado durante a pesquisa nas bases de dados. Mesmo em bases como SciELO e PePSIC, nas quais é possível consultar periódicos publicados no Brasil, não houve indicação de trabalho que utilizasse o termo descritor apego ao lugar, observando-se apenas uma ocorrência de artigo contendo a expressão no campo resumo. A falta de produção nacional com atenção específica a laços afetivos com lugares denuncia um descompasso de interesses entre nações, compreensível, em certa medida, pelo fato de a produção internacional mais expressiva sobre o tema ser notadamente recente.

\section{Referências}

Ainsworth, M. D. S. (1989). Attachments beyond infancy. American Psychologist, 44 (4), 709-716. Retrieved on April 19,2009, from <http://psycnet.apa.org/journals/amp/44/ 4/709/>.

Allard, T., Babin, B. J., \& Chebat, J. C. (2009). When income matters: customers evaluation of shopping malls' hedonic and utilitarian orientations. Journal of Retailing and Consumer Services, 16 (1), 40-49. Retrieved on April 19, 2009, from <http://www.sciencedirect.com/science/ article/pii/S0969698908000416>.

Bonaiuto, M., Carrus, G., Martorella, H., \& Bonnes, M. (2002). Local identity processes and environmental attitudes in land use changes: the case of natural protected areas. Journal of Economic Psychology, 23, 631-653.

arte

- A observação de vestígios ambientais e o mapeamento comportamental são técnicas de observação valiosas nos estudos pessoa-ambiente (Pinheiro, Elali \& Fernandes, 2008). A primeira propõe o estudo de traços do comportamento pela observação sistemática das marcas deixadas em um local, ainda que não se presencie o comportamento propriamente dito. A segunda se refere à tarefa de associar comportamentos determinados a porções específicas do espaço, representadas em um mapa. 
Bowlby, J. (1997). Formação e rompimento dos laços afetivos (3a ed.). São Paulo: Martins Fontes. (Originalmente publicado em 1979).

Brown, B. B., \& Perkins, D. D. (1992). Disruption in place attachment. In I. Altman \& S. M. Low (Eds.), Place attachment (pp.279-304). New York: Plenum Press.

Brown, B. B., Perkins, D. D., \& Brown, G. (2003). Place attachment in a revitalizing neighborhood: individual and block levels of analysis. Journal of Environmental Psychology, 23 (3), 259-271. Retrieved on April 19, 2009, from <http://www.sciencedirect.com/science/article/pii/ S0272494402001172>.

Brown, B. B., Perkins, D. D., \& Brown, G. (2004). Incivilities, place attachment and crime: block and individual effects. Journal of Environmental Psychology, 24 (3), 359-371. Retrieved on April 19, 2009, from <http://www. sciencedirect.com/science/article/pii/S027249440 4000192>.

Brown, G., Brown, B. B., \& Perkins, D. D. (2004). New housing as neighborhood revitalization: place attachment and confidence among residents. Environment and Behavior, 36 (6), 749-775. Retrieved on April 19, 2009, from <http://eab.sagepub.com/content/36/6/749.short>.

Brown, G. G., Reed, P., \& Harris, C. C. (2002). Testing a placebased theory for environmental evaluation: an Alaska case study. Applied Geography, 22 (1), 49-76. Retrieved on April 19, 2009, from <http://www.sciencedirect.com/ science/article/pii/S0143622801000194>.

Buijs, A. E. (2009). Public support for river restoration: a mixed-method study into local residents' support for and framing of river management and ecological restoration in the Dutch floodplains. Journal of Environmental Management, 90 (8), 2680-2689. Retrieved on April 19, 2009, from <http://www. sciencedirect.com/ science/article/pii/S0301479709000541>.

Elali, G. A. (1997). Psicologia e Arquitetura: em busca do locus interdisciplinar. Estudos de Psicologia, 2 (2), 349-362.

Giuliani, M. V. (2003). Theory of attachment and place attachment. In M. Bonnes, T. Lee \& M. Bonaiuto (Eds.), Psychological theories for environmental issues (pp.137-170). Aldershot: Ashgate.

Giuliani, M. V. (2004). O lugar do apego nas relações pessoas-ambiente. In E. T. O. Tassara, E. P. Rabinovich \& M. C. Guedes (Eds.), Psicologia e ambiente (pp.89-106). São Paulo: Educ.

Gross, M. J., \& Brown, G. (2006). Tourism experiences in a lifestyle destination setting: the roles of involvement and place attachment. Journal of Business Research, 59 (6), 696-700. Retrieved on April, 2009, from <http://www. sciencedirect.com/science/article/pii/S014829630 6000208>

Gross, M. J., \& Brown, G. (2008). An empirical structural model of tourists and places: progressing involvement and place attachment into tourism. Tourism Management, 29 (6), 1141-1151. Retrieved on April 19, 2009, from <http:// www.sciencedirect.com/science/article/pii/S026151 7708000344>
Günther, H., Elali, G. A., \& Pinheiro, J. Q. (2008). A abordagem multimétodos em estudos pessoa-ambiente: características, definições e implicações. In J. Q. Pinheiro \& H. Günther (Eds.), Métodos de pesquisa nos estudos pessoaambiente (pp.369-396). São Paulo: Casa do Psicólogo.

Hailu, G., Boxall, P. C., \& McFarlane, B. L. (2005). The influence of place attachment on recreation demand. Journal of Economic Psychology, 26 (4), 581-598. Retrieved on April 19, 2009, from <http://www.sciencedirect. com/science/ article/pii/S016748700400114X>.

Hwang, S. N., Lee, C., \& Chen, H. J. (2005). The relationship among tourists' involvement, place attachment and interpretation satisfaction in Taiwan's national parks. Tourism Management, 26 (2), 143-156. Retrieved on April 19, 2009, from <http://www.sciencedirect. com/science/ article/pii/S026151770300236X>.

Jorgensen, B. S., \& Stedman, R. C. (2006). A comparative analysis of predictors of sense of place dimensions: attachment to, dependence on, and identification with lakeshore properties. Journal of Environmental Management, 79 (3), 316-327.

Kelly, G., \& Hosking, K. (2008). Nonpermanent residents, place attachment, and "sea change" communities. Environment and Behavior, 40 (4), 575-594. Retrieved on April 19, 2009, from <http://eab.sagepub. com/content/ 40/4/575.abstract?ijkey=d42e8c16b8 cff8b6166db3 99c97deda092488cea\& keytype2=tf_ ipsecsha $>$.

Lewicka, M. (2005). Ways to make people active: the role of place attachment, cultural capital, and neighborhood ties. Journal of Environmental Psychology, 25 (4), 381-395. Retrieved on April 19, 2009, from <http://www. sciencedirect.com/science/article/pii/S027249 4405000691>.

Manzo, L. C., \& Perkins, D. D. (2006). Finding common ground: the importance of place attachment to community participation and planning. Journal of Planning Literature, 20 (4), 335-350. Retrieved on April 19, 2009, from <http://jpl.sagepub.com/content/20/4/335. abstract>.

Pinheiro, J. Q. (2003). Psicologia Ambiental brasileira no início do século XXI: sustentável? In O. H. Yamamoto \& V. V. Gouveia (Eds.), Construindo a psicologia brasileira:desafios da ciência e prática psicológica (pp.279-313). São Paulo: Casa do Psicólogo.

Pinheiro, J. Q., Elali, G. A., \& Fernandes, O. S. (2008). Observando a interação pessoa-ambiente: vestígios ambientais e mapeamento comportamental. In J. Q. Pinheiro \& H. Günther (Eds.), Métodos de pesquisa nos estudos pessoa-ambiente (pp. 75-104). São Paulo: Casa do Psicólogo.

Proshansky, H. M., Fabian, A. K., \& Kaminoff, R. (1983). Placeidentity: physical world socialization of the self. Journal of Environmental Psychology, 3, 57-83. Retrieved on April 19,2009, from < http://www.sciencedirect. com/science/ article/pii/S0272494483800218>

Rosenbaum, M. S., Ward, J., Walker, B. A., \& Ostrom, A. L. (2007). A cup of coffee with a dash of love: an investigation of commercial social support and third-place attachment. Journal of Service Research, 10, 43-59. 
Ryan, R. L. (2005). Exploring the effects of environmental experience on attachment to urban natural areas. Environment and Behavior, 37 (1), 3-42. Retrieved on April 19, 2009, from <http://eab.sagepub.com/content/37/1/ 3>.

Sève, L. (1979). Marxismo e teoria da personalidade (Vol. 1). Lisboa: Livros Horizonte.

Shumaker, S. A., \& Taylor, R. B. (1983). Toward a clarification of people-place relationships: a model of attachment to place. In N. R. Feimer \& E. S. Geller (Eds.), Environmental psychology: directions and perspectives (pp.119-251). New York: Praeger.

Speller, G. M. (2005). A importância da vinculação ao lugar. In L. Soczka (Ed.), Contextos humanos epsicologia ambiental (pp.133-167). Lisboa: Fundação Calouste Gulbenkian.

Valera, S. (1996). Psicologia ambiental: bases teóricas y epistemológicas. In L. Iñiguez \& E. Pol (Eds.), Cognición, representación y apropiación del espacio (pp.1-14). Barcelona: Universidad de Barcelona Publicacions.

Wakefield, S. E. L., Elliott, S. J., Cole, D. C., \& Eyles, J. D. (2001). Environmental risk and (re)action: air quality, health, and civic involvement in an urban industrial neighbourhood. Health \& Place, 7 (3), 163-177. Retrieved on April 19, 2009, from <http://www.sciencedirect.com/science/ article/pii/S1353829201000065>.

Walker, A. J., \& Ryan, R. L. (2008). Place attachment and landscape preservation in rural New England: a maine case study. Landscape and Urban Planning, 86 (2), 141-152. Retrieved on April 19, 2009, from <http://www. sciencedirect.com/science/article/pii/S01692046080 00315>.

Yuksel, A., Yuksel, F., \& Bilim, Y. (2010). Destination attachment: effects on customer satisfaction and cognitive, affective and conative loyalty. Tourism Management, 31 (2), 274-284. Retrieved on April 19, 2009, from <http:// www.sciencedirect.com/science/article/pii/S02615177 09000508>

Recebido em: 6/5/2010

Versão final em: 28/10/2011

Aprovado em: 9/3/2012 